\title{
Ionising radiation from diagnostic $x$ rays in very low birthweight babies
}

\author{
P M Sutton, R J Arthur, C Taylor, M D Stringer
}

\begin{abstract}
Aim-To quantify the exposure of very low birthweight neonates to ionising radiation from diagnostic $x$-rays.

Methods-Retrospective analysis was made of all radiographs performed over 18 months in an integrated special care baby unit and regional neonatal surgical unit in a large teaching hospital of surviving inborn babies of very low birthweight $(<1500 \mathrm{~g})$ admitted to the unit.

Results-Fifty five VLBW neonates were treated for a total of 3296 days and received 498 radiographs (median 5 per infant). The mean effective radiation dose was $0.04 \mathrm{mSv}$ and the maximum for one infant was $0.54 \mathrm{mSv}$.

Conclusions-The radiation burden in this group of neonates is low and the benefits of diagnostic radiographs far outweigh any potential radiation risks.

(Arch Dis Child Fetal Neonatal Ed 1998;78:F227-F229)
\end{abstract}

Keywords: radiographs; ionising radiation; very low birthweight

Very low birthweight infants are prone to a variety of medical and surgical problems such as respiratory distress syndrome and necrotising enterocolitis, and so they are exposed to a relatively large number of radiographs. The harmful effects of radiation are greater when exposure occurs early in life ${ }^{1}$ and because of their size, a relatively large area of the infant may be irradiated and it is more difficult to shield radiosensitive organs. Both medical staff and parents of small premature babies often question the safety of multiple radiographs, but there is very little published information on this subject. Our aim was to quantify exposure to ionising radiation in a specific group of premature, very low birthweight neonates.

Department of Paediatric Surgery, Clarendon Wing, Leeds General Infirmary, Leeds LS2 9NS

P M Sutton

M D Stringer

Department of Paediatric Radiology

R J Arthur

Department of Medical Physics, Cookridge Hospital, Leeds C Taylor

Correspondence to: Mr M D Stringer.

Accepted 3 November 1997 infants referred from other hospitals and those that died or were discharged to other units for continuing inpatient care. We considered a retrospective analysis to be essential so as to avoid influencing current radiographic practice.

We recorded demographic details including gestation, birthweight, discharge weight, duration of admission in days and all medical or surgical diagnoses for each infant. Each radiograph was assessed with respect to the date, type of radiograph, and exposure details. The exposed area of each radiograph was measured to the nearest $\mathrm{mm}^{2}$. Projection and exposure details were cross referenced to independent filed records completed by the radiographers at the time of each radiograph. Three infants underwent a radiological screening procedure. At the time of this study the screening equipment was not fitted with a dose-area product meter and as the calculation of imparted energy and absorbed dose would not therefore be accurate, these investigations were excluded from the final analysis.

Two different types of radiographic equipment were in use during the study. During the first five months, all radiographs were taken using an AMX4 set (IGE Medical Systems Ltd). Subsequently, the set was changed to a CD38S (Picker). The outputs of these two sets were measured over a range of tube voltages $(\mathrm{kV})$. A focus to film distance (FFD) of 1 metre was chosen for these measurements (table 1).

Using the data collected, the surface entrance dose for each radiograph, the imparted energy, and the mean absorbed dose for each infant can be calculated using the following formulae:

- Surface entrance dose ( $\mu G y$ )

Output $\times$ mAs $\times$

Entrance dose $(\mu \mathrm{Gy})=\frac{\text { Backscatter factor }}{(\mathrm{FSD})^{2}}$

$\mathrm{FSD}=$ focus to skin distance measured at 0.91 $\mathrm{m}$ for a typical neonate. A backscatter factor of 1.1 was applied as this figure has already been calculated and validated in neonatal radiology. ${ }^{2}$

- Imparted energy (mf)

Imparted energy $=$ dose in air at FFD $\times$ area of field $\left(\mathrm{cm}^{2}\right) \times$ conversion factor

$\mathrm{FFD}=$ Focus to film distance $(1$ metre $)$.

$$
\text { Dose in air at FFD }=\frac{\text { output } \times \text { mAs }}{(\mathrm{FFD})^{2}}
$$

As there are no published tables of conversion factors for low birthweight neonates, we based our conversion factor of $4 \times 10^{-3} \mathrm{~mJ} \mathrm{mGy}^{-1}$ $\mathrm{cm}^{-2}$ on the work of Chapple, Faulkner and Hunter. $^{2}$ 
Table 2 Medical conditions complicating prematurity in study group

\begin{tabular}{lc}
\hline Diagnosis & $\begin{array}{l}\text { Number of neonates } \\
\text { with diagnosis }\end{array}$ \\
\hline Respiratory distress syndrome & 33 \\
Chronic lung disease & 19 \\
Persistent ductus arteriosus & 6 \\
Necrotising enterocolitis & 6 \\
Intraventricular haemorrhage & 14 \\
Congenital malformations & 2 \\
Other problems & 4 \\
\hline
\end{tabular}

- Mean absorbed dose ( $m G y$ )

Absorbed radiation dose to the whole body $(\mathrm{mGy})=\frac{\text { energy imparted }(\mathrm{mJ})}{\text { Mass }(\mathrm{kg})}$

To simplify calculation we have assumed that linear growth occurs from birth to time of discharge home and that as a result of the small size of these neonates all radiosensitive tissues would be irradiated equally. Our estimate of effective dose is based on this assumption, though clearly growth is not exactly linear in these patients.

\section{Results}

During the study period 126 VLBW infants were delivered at Leeds General Infirmary. Twenty infants died and 51 were discharged to other hospitals, leaving 55 infants available for analysis. This cohort had a mean gestational age of 28.8 weeks (range 24-34 weeks), a mean birthweight of $1.11 \mathrm{~kg}$ (range $0.54-1.48 \mathrm{~kg}$ ) and a mean duration of hospital stay of 60 days (range 4-239 days). Forty four neonates had

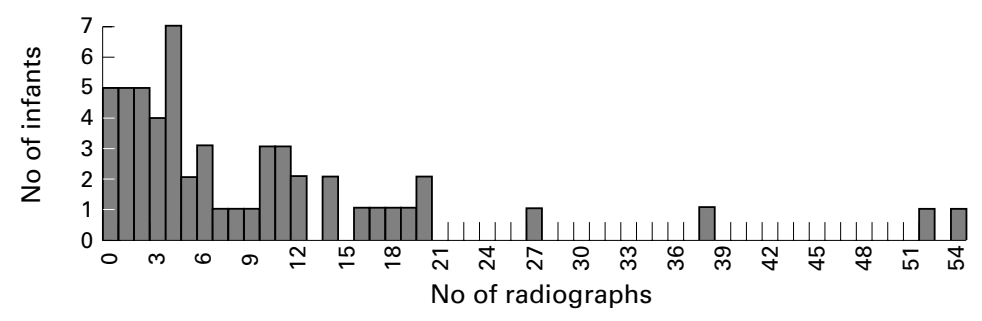

Figure 1 Frequency distribution of number of radiographs per infant.

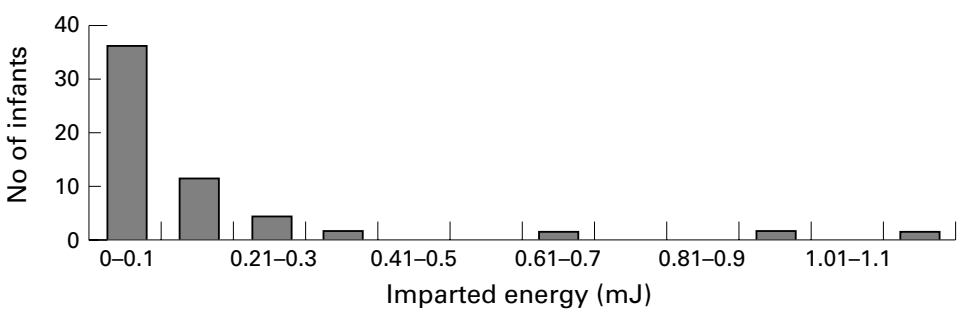

Figure 2 Frequency distribution of total energy imparted (mf) per infant.

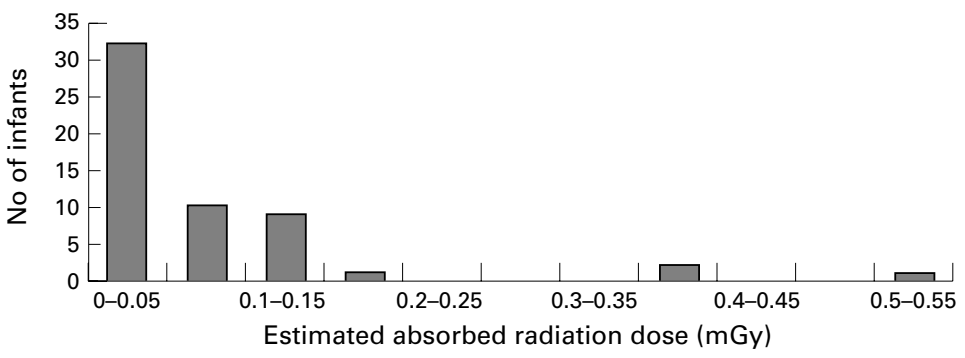

Figure 3 Frequency distribution of estimated absorbed radiation dose per infant.
Table 3 Number of $x$ ray pictures performed and mean surface entrance dose for each examination

\begin{tabular}{lcl}
\hline Type of $x$ ray & $\begin{array}{l}\text { Number } \\
\text { performed }\end{array}$ & $\begin{array}{l}\text { Mean surface } \\
\text { entrance dose }(\mu G y)\end{array}$ \\
\hline Chest & $333(67 \%)$ & 31.9 \\
Abdominal & $77(15 \%)$ & 31.8 \\
Chest and abdomen & $34(7 \%)$ & 43.7 \\
Upper limb & $46(9 \%)$ & 39.8 \\
Lower limb & $5(1 \%)$ & 36.9 \\
Skull & $3(1 \%)$ & 63 \\
\hline
\end{tabular}

major medical problems, largely related to prematurity (table 2). Of the 33 with respiratory distress syndrome, positive pressure ventilation was required for a mean of 9.2 days (range 12 hours to 67 days).

A total of 498 radiographs and three radiological contrast procedures was performed. Information from $11(2.2 \%)$ radiographs was incomplete and these were excluded from subsequent analysis. The number of radiological procedures per child ranged from 0 to 54 with a median of 5 (mean 9.1) (fig $1)$.

The mean surface entrance dose per film was 33.1 $\mu \mathrm{Gy}$ (range 15.3-73.9). The main cause of this variation was the type of examination (table 3). The maximum total energy imparted to an individual neonate was $1.11 \mathrm{~mJ}$; this infant was exposed to 52 radiographs over a period of 210 days and had the highest absorbed radiation dose (0.54 mGy). The median total imparted energy and absorbed dose for each infant was $0.06 \mathrm{~mJ}$ (mean 0.12 $\mathrm{mJ}$ ) and $0.04 \mathrm{mGy}$ (mean $0.07 \mathrm{mGy}$ ). Figures 2 and 3 show the frequency distributions of imparted energy and absorbed radiation for all patients.

\section{Discussion}

Parents and clinicians are often concerned about the potential hazard of radiation in children. This problem is highlighted in very low birthweight babies who spend a long time in hospital and are prone to complications of prematurity that necessitate radiographic diagnosis and monitoring. Children seem to be more sensitive than adults to the carcinogenic effects of radiation. ${ }^{3}$ Reports of the potential link between low level environmental radiation exposure and the development of leukaemia have highlighted these concerns in recent years. Since the inception of our study, one report of radiation exposure in neonates has been published, ${ }^{2}$ but in general there is little information on this subject. We deliberately conducted a retrospective study so as to avoid any bias in the results due to greater awareness of radiation risks leading to an alteration in our normal radiographic practice.

VLBW infants in our unit received a median of five radiographs equivalent to less than one radiograph a week. Figures 1 to 3 show a markedly skewed distribution, with only a few neonates exposed to relatively large numbers of radiographs. It is difficult to estimate the potential increased risk of a fatal cancer from $x$-ray exposure as a neonate. The risk of fatal childhood cancer (to age 15) associated with in utero exposure to ionising radiation is estimated 
to be $3 \times 10^{-5} \mathrm{mSv}^{-1}$. As the infants in our study were all premature and of very low birthweight, this figure is a useful guide. Even at the most extreme level (the neonate with the effective radiation dose of $0.54 \mathrm{mSv}$ ) the amount of radiation received was very low. By comparison, an individual would receive a similar radiation dose in three months from natural background radiation. ${ }^{5}$ The increased risk of fatal childhood cancer for this infant is estimated at 1 in 60000 ; this risk is hugely outweighed by the overall risk of mortality in VLBW infants. Our results compare well with those of Chapple et al, who studied a less well defined group of neonates in a special care baby unit in Newcastle and estimated the mean entrance dose per radiograph to be $55 \mu \mathrm{Gy}$ and the mean absorbed radiation dose to be $0.04 \mathrm{mGy} .^{2}$

We accept that these figures only represent estimates of the radiation burden in these infants, particularly as various assumptions have to be made in the calculations. However, exact measurement using a dose-area product meter is impractical in this group of neonates who are usually radiographed in their incubators using mobile $x$-ray machines. For several reasons, the radiation dose may have been underestimated in this study. Firstly, infants discharged from our unit to other hospitals were excluded from analysis and these babies may have had a more complicated neonatal course requiring more radiographs. Secondly, many premature neonates such as those with chronic lung disease will have further radiological investigations throughout childhood. Thirdly, it was not possible to take account of the need for repeat films (approximately $5 \%$ in our unit) and we excluded three screening procedures from the analysis. Finally our assump- tion that the imparted energy was evenly distributed over the whole body may be incorrect. In calculating effective dose it may be more accurate to assume that the radiation field was confined to the trunk which constitutes approximately $50 \%$ of a neonate's mass and that all radiosensitive tissues in the trunk are irradiated uniformly. This would effectively double our estimate of absorbed tissue dose used to calculate the effective dose, thereby doubling the effective dose.

Despite these limitations, we believe that the methodology used provides a sufficiently accurate estimation to conclude that the amount of radiation received by VLBW neonates is generally low. However, there is clearly no room for complacency. Whilst radiographic examinations undoubtedly contribute to the care of these infants it is essential that each request should be carefully considered to ensure that the examination is justified and likely to influence clinical management. Furthermore, the examination should be performed at the lowest achievable dose, attending to details of technique such as exposure factors and collimation.

We acknowledge the care and expertise of our neonatal medical, nursing and radiographic colleagues.

1 International Commission on Radiological Protection. Risks associated with ionising radiations. Annals of the International Commission on Radiological Protection 1991;22:9-102.

2 Chapple CL, Faulkner K, Hunter EW. Energy imparted to neonates during $\mathrm{X}$-ray examinations in a special care baby unit. Br F Radiol 1994;67:366-70.

3 Boice JD. Cancer following irradiation in childhood and adolescence. Med Ped Oncol 1996;Supp1 1:29-34.

4 National Radiological Protection Board. Estimates of late radiation risk to the UK population. Documents of the radiation risk to the UK population. Docum
$N R P B, 4: 15-157$. London: HMSO, 1993.

5 Hughes JS, O'Riordan MC. Radiation Exposure of the UK Population - 1993 Review. NRPB-R263. London: HMSO 